\title{
iTRAQ-based proteomic analysis of the interaction of A549 human lung epithelial cells with Aspergillus fumigatus conidia
}

\author{
XIAOWEI ZHANG ${ }^{1}$, DAN HE $^{1}$, SONG GAO $^{1,2}$, YUNYUN WEI $^{1}$ and LI WANG ${ }^{1}$ \\ ${ }^{1}$ Department of Pathogenobiology, Jilin University Mycology Research Center, College of Basic Medical Sciences, \\ Jilin University, Changchun, Jilin 130021; ${ }^{2}$ Beijing Zhongkai Tiancheng Bio-technology Co. Ltd., Beijing 100176, P.R. China
}

Received January 28, 2020; Accepted August 12, 2020

DOI: $10.3892 / \mathrm{mmr} .2020 .11582$

\begin{abstract}
Severe invasive aspergillosis infection occurs when human immune function is impaired. The interaction between Aspergillus fumigatus (A.fumigatus) conidia and type II lung epithelial cells serves an important role in disease progression. The present study compared the proteomes of A549 human lung epithelial cells with and without $A$. fumigatus infection. Gene Ontology (GO), Kyoto Encyclopedia of Genes and Genomes (KEGG) and protein interaction analyses were performed, and differential protein expression was verified by western blotting and reverse transcription-quantitative PCR (RT-qPCR). In addition, the RNA interference method, an internalization assay and ELISA were performed. Isobaric tags for relative and absolute quantification analysis detected a total of 1,582 proteins, from which 111 proteins with differential expression were obtained (fold change $>1.5$ or $<0.75$ ). Among them, 18 proteins were upregulated and 93 proteins were downregulated in A549 cells challenged with A. fumigatus. GO and KEGG analyses revealed that the altered proteins were mainly involved in biological functions, such as cell metabolism, synthesis, the cellular stress response, metabolic pathways and pyruvate metabolism. N-myc downstream-regulated gene 1 (NDRG1) expression was upregulated 1.88-fold, while CD44 expression was downregulated 0.47-fold following A. fumigatus infection. The expression levels of specific proteins were verified by western blotting and RT-qPCR . The internalization efficiency was affected by NDRGl gene silencing. The secretion of IL- 6 and IL- 8 was affected when CD44 was inhibited. These results indicated that A.fumigatus affects lung epithelial cell metabolism and biological synthetic functions. A number of novel molecules, including NDRG1 and $\mathrm{CD} 44$, were found to be related to A.fumigatus infection.
\end{abstract}

Correspondence to: Professor Li Wang, Department of Pathogenobiology, Jilin University Mycology Research Center, College of Basic Medical Sciences, Jilin University, 126 Xinmin Street, Changchun, Jilin 130021, P.R. China

E-mail: wli99@jlu.edu.cn

Key words: Aspergillus fumigatus, fungal infection, isobaric tags for relative and absolute quantification analysis, proteomic analysis, lung epithelial cells

\section{Introduction}

The conidia of Aspergillus fumigatus (A. fumigatus), a common fungus, are $\sim 2-3 \mu \mathrm{m}$ in size and easily spread by wind (1). Humans can inhale 100s of conidia every day, and conidia can also penetrate into the alveoli via mucociliary clearance in lung (2). Hosts with healthy immune functions can phagocytose or kill conidia via alveolar macrophages, thus infections rarely occur. However, once the body's immune function is impaired, invasive pulmonary aspergillosis (IPA) occurs and gradually spreads to the kidneys, brain and other organs; IPA exhibits a mortality rate as high as $50-90 \%$ (3). A. fumigatus is the second leading cause of invasive fungal diseases worldwide (4).

The lungs are the initial organs that the A.fumigatus would first come into contact with. Airway epithelial cells (including lung epithelial cells and tracheal epithelial cells) are the site of the initial process of A.fumigatus conidia. Alveolar epithelial cells are composed of alveolar epithelial type I and type II cells, and inhaled conidia mainly interact with type II cells, which maintain the alveolar space (5). An In vitro study has demonstrated that A. fumigatus conidia first adhere to the surface of type II lung epithelial cells and are then endocytosed by lung epithelial cells, which triggers an immune response and respiratory burst (6). The surviving conidia remain and germinate into hyphae, causing infection under suitable conditions (7). The interaction between A. fumigatus conidia and type II lung epithelial cells serves an important role in disease progression (6). The present study aimed to explore the molecular mechanism of the interaction between them.

Isobaric tags for relative and absolute quantitation (iTRAQ) are an isotopically labeled relative and absolute quantification technique (8). Combined with liquid chromatography-mass spectrometry (LC/MS), it has advantages over traditional protein quantification. iTRAQ is able to mark most of the proteins in a sample, and the labeling process is simple, more timesaving and more accurate. At the same time, this method enables quantitative comparison of proteins in multiple samples in the same test to obtain a large amount of protein information.

In the present study, A549 cells were explored as an in vitro cell model. The proteomes of A549 type II lung epithelial cells with and without $A$. fumigatus challenge were compared using the isobaric tags for relative and absolute 
quantification (iTRAQ) technique. Additionally, the functions of significantly differentially expressed proteins, such as N-myc downstream-regulated gene 1 (NDRG1) and CD44, were discussed.

\section{Materials and methods}

Culture of A. fumigatus strain IFM40808 and A549 type II lung epithelial cells. A. fumigatus strain IFM40808 was provided by the Medical Mycology Research Center of Chiba University (Chiba, Japan). The strain was cultured on potato glucose medium (Difco; BD Biosciences) for 7 days at $37^{\circ} \mathrm{C}$ with $5 \% \mathrm{CO}_{2}$. The conidia suspension was collected after being flushed with sterile $0.05 \%$ Tween- 20 in 50 mM PBS $(\mathrm{pH}$ 7.4) and the conidia concentration was then adjusted to $2 \times 10^{7} \mathrm{cfu} / \mathrm{ml}$ with RPMI-1640 medium (Gibco; Thermo Fisher Scientific, Inc.).

A549 human type II lung epithelial cells were purchased from American Type Culture Collection. Cells were cultured in RPMI-1640 medium containing 10\% fetal bovine serum (Gibco; Thermo Fisher Scientific, Inc.), $50 \mathrm{IU} / \mathrm{ml}$ penicillin and $50 \mu \mathrm{g} / \mathrm{ml}$ streptomycin at $37^{\circ} \mathrm{C}$ with $5 \% \mathrm{CO}_{2}$.

Co-incubation of A. fumigatus conidia and A549 lung epithelial cells. The A549 cell concentration was adjusted to $2 \times 10^{6}$ cells $/ \mathrm{ml}$ and the cells were transferred to dishes in medium without fetal bovine serum, following which culture was continued for 16-18 h. The A549 cells were infected by A.fumigatus conidia at an MOI of 10 (i.e., 10 conidia to 1 cell) and cocultured for $6 \mathrm{~h}$ after infection in serum-free medium at $37^{\circ} \mathrm{C}$ with $5 \% \mathrm{CO}_{2}$.

Protein extraction, digestion and labelling with iTRAQ reagents. The supernatant following co-incubation was aspirated and washed with pre-cooled PBS at $4^{\circ} \mathrm{C}$. The proteins were extracted in accordance with the methods of Vuong et al (9) and She et al (10). Cell total proteins were extracted by grinding with liquid nitrogen and suspending in lysis buffer [ $8 \mathrm{M}$ urea, $1 \%$ Triton X-100, $65 \mathrm{mM}$ dithiothreitol (DTT) Sigma-Aldrich; Merck KGaA) and $0.1 \%$ protease inhibitor cocktail (Sigma-Aldrich; Merck KGaA). Proteins were then sonicated on ice five times ( 5 mins for one time, and $30 \mathrm{sec}$ on, $30 \mathrm{sec}$ off) for five cycles using ultrasound sonicator (Ningbo Scientz Biotechnology Co., Ltd.). Lysates were centrifuged at $5,000 \mathrm{xg}$ at $4^{\circ} \mathrm{C}$ for $3 \mathrm{~min}$ and the supernatant was collected. The proteins were precipitated with cold 15\% Trichloroacetic acid (Thermo Fisher Scientific, Inc.) for $2 \mathrm{~h}$ at $-20^{\circ} \mathrm{C}$. The precipitate were collected via centrifugation at $15,000 \mathrm{x}$ g for $10 \mathrm{~min}$ at $4^{\circ} \mathrm{C}$ and rinsed three times with cold acetone. The air-dried protein pellet was dissolved in lysis buffer $(8 \mathrm{M}$ urea, $100 \mathrm{mM}$ Triethylamine-carbonic acid buffer, $\mathrm{pH}$ 8.0) and the protein concentration was determined using a 2-D Quant kit (Cytiva), and then adjusted to $2 \mathrm{mg} / \mathrm{ml}$.

Proteolysis and peptide labelling were carried out in accordance with the methods of She et al (10) and Pizzatti et al (11). For proteolysis, the protein solution was treated with $10 \mathrm{mM}$ DTT for $1 \mathrm{~h}$ at $37^{\circ} \mathrm{C}$ and $20 \mathrm{mM}$ iodoacetamide (Sigma-Aldrich; Merck KGaA) for $45 \mathrm{~min}$. The solution was diluted with TEAB to obtain a final solution containing $1 \mathrm{M}$ urea. Then, the solution was digested with trypsin (dilution, 1:50) overnight. Trypsin (Promega Corporation) was added at 1:50 for digestion overnight and 1:100 for a second 4 h-digestion. Following enzymatic hydrolysis, the obtained peptides were desalted using a Strata-X C18 solid phase extraction column (Phenomenex Inc.). After vacuum freeze-drying, the two samples were labelled according to the instructions of the iTRAQ Reagent8-Plex kit (AB Sciex Pte., Ltd.).

Inspection by $L C / M S$. The peptides from the two groups were separated using a Waters 600E reversed-phase high-performance liquid chromatography system with an Agilent 300 Extend C18 column ( $5 \mu \mathrm{m}$ particle size, $4.6 \mathrm{~mm}$ ID, $250 \mathrm{~mm}$ length; Agilent Technologies,Inc.). The column was maintained at room temperature and the flow rate was $1 \mathrm{ml} / \mathrm{min}$. Briefly, the peptide mixture was first separated with a gradient of 2-60\% acetonitrile in $10 \mathrm{mM}$ ammonium bicarbonate $(\mathrm{pH} 10)$, over $80 \mathrm{~min}$ into 80 fractions. The fractions were combined into eight components and vacuum-dried. The analysis was performed using an LTQ Orbitrap Elite LC/MS (Thermo Fisher Scientific, Inc.) equipped with a Dionex UltiMate 3000 nano-UPLC system (Thermo Fisher Scientific, Inc.). A guard column (C18 PepMap 100, $300 \mu \mathrm{m}$ x 1 mm, $5 \mu \mathrm{m}, 100 \AA$ A) and an analytical column (Acclaim PepMap C18, $15 \mathrm{~cm} \times 75 \mu \mathrm{m}$, $2 \mu \mathrm{m}, 100$ Å; Dionex;Thermo Fisher Scientific, Inc.) were applied. Mobile phase A (ultra-pure water, $0.1 \%$ formic acid; Honeywell Fluka) at a gradient of $\sim 6-44 \%$ was used during the run, followed by mobile phase B [98\% acetonitrile (Thermo Fisher Scientific, Inc.), $0.1 \%$ formic acid] at a gradient of 44-98\%. After one cycle had ended, the column was equilibrated with $98 \%$ mobile phase B followed by $6 \%$ mobile phase A. The column was maintained by equilibrating with $6 \%$ mobile phase A for $5 \mathrm{~min}$ before the next injection. The technical parameters were in accordance with the settings of Zhang et al (12). The parameters for MS1 were set as follows: Cation-positive mode; Orbitraq resolution: 60,000; scan range, $400 \mathrm{~m} / \mathrm{z}$. The parameters for MS2 were set as: Orbitrap resolution: 15,000; activation type, High Energy Collision Dissociation; normalized collision energy setting, 35\%. The working conditions were: Nitrogen temperature $350^{\circ} \mathrm{C}$ at a flow rate of $12 \mathrm{l} / \mathrm{min}$, nebulizer pressure $60 \mathrm{psi}$ and electrospray capillary voltage $+3,000 \mathrm{~V}$.

Protein database alignment and relative quantification by $i T R A Q$. The obtained raw protein data were aligned using the SEQUEST engine built into Proteome discoverer software version 2.0 (Thermo Fisher Scientific, Inc.). The Human International Protein Index (IPI) protein database (IPI v3.87; ftp://ftp.ebi.ac.uk/pub/databases/IPI; a total of 91,464 records) was referenced for analysis.

Function analysis of differentially expressed proteins. Data on differentially expressed proteins were entered into the Database for Annotation, Visualization and Integrated Discovery (version 6.7; http://david.abcc.ncifcrf.gov/). Gene Ontology (GO; http://wego.genomics.org.cn/cgi-bin/wego/index.pl) and Kyoto Encyclopaedia of Genes and Genomes (KEGG; $\mathrm{http} / / / \mathrm{www}$.genome.jp/kegg/) analyses were applied for functional prediction of the obtained differentially expressed proteins. The obtained GO terms and KEGG pathways were 
Table I. Primers for reverse transcription-quantitative PCR.

\begin{tabular}{llll}
\hline Gene & Forward primer sequence (5'-3') & Reverse primer sequence (5'-3') & Amplicon size(bp) \\
\hline$N D R G 1$ & GTCCTTATCAACGTGAACCCTT & GCATTGGTCGCTCAATCTCCA & 250 \\
$S L K$ & CAATGCGAGTGCTGCTAAAAAT & CGGATGGGTTTGTTGGAATCA & 184 \\
CD44 & CTGCCGCTTTGCAGGTGTA & CATTGTGGGCAAGGTGCTATT & 109 \\
GAPDH & TGTGGGCATCAATGGATTTGG & ACACCATGTATTCCGGGTCAAT & 116 \\
\hline
\end{tabular}

NDRG1, N-myc downstream-regulated 1; SLK, STE20-like serine/threonine-protein kinase.

considered enriched in the differentially expressed proteins when $\mathrm{P}<0.05$ as determined by tests of statistical significance. Protein interaction network analysis of differentially expressed proteins was performed using the protein interaction network online database Search Tool for the Retrieval of Interacting Genes/Proteins (STRING; version 11.0; http://string.embl.de). The graphs were visualized using Cytoscape 2.8 (http://www. cytoscape.org/) (13).

Western blot analysis. The iTRAQ results obtained from the infected group and the control group were verified by western blotting. Following infection with A. fumigatus conidia at an MOI of 10 for $6 \mathrm{~h}$, the infected A549 cells were collected. The protein in cells was extracted using RIPA buffer (Beyotime Institute of Biotechnology) and evaluated using the BCA Protein Assay kit (Beyotime Institute of Biotechnology). Equal amounts (40 ng) of protein were separated via 12\% SDS-PAGE and transferred to PVDF membrane (EMD Millipore). The membrane was then blocked with $5 \%$ nonfat milk powder in PBS with $0.1 \%(\mathrm{v} / \mathrm{v})$ Tween (PBST) for $1 \mathrm{~h}$ at room temperature, followed by rabbit anti-human primary antibodies (dilution, 1:500) against N-myc downstream-regulated gene 1 (NDRG1; cat. no. 9485S, Cell Signaling Technology, Inc.), isoform 1 of STE20-like serine/threonine-protein kinase (SLK; cat. no. 41255S; Cell Signaling Technology, Inc.) and CD44 (cat. no. 37259S; Cell Signaling Technology, Inc.), and the internal reference protein GAPDH (cat. no. AF7021; dilution, 1:1,000; Affinity Biosciences) at $4^{\circ} \mathrm{C}$ overnight. The membrane was incubated with goat anti-rabbit IgG (H+L)-HRP (cat. no. S0001; dilution, 1:5,000; Affinity Biosciences) secondary antibody at room temperature for $1 \mathrm{~h}$. The expression levels of target proteins were detected by ECL (PerkinElmer, Inc.). Proteins were observed and images were captured with a chemiluminescence gel imager. Grayscale analysis was performed using ImageJ software (Version 1.52a; National Institutes of Health).

RNA extraction and reverse transcription-quantitative PCR (RT-qPCR). A549 cells $\left(1 \times 10^{6}\right)$ with or without A. fumigatus infection were harvested and the total RNA extraction performed with TRIzol $^{\circledR}$ (Invitrogen; Thermo Fisher Scientific, Inc.). The total RNA (1 $\mu \mathrm{g})$ was then reverse transcribed into cDNA using the TransCript One-step gDNA Removal and cDNA Synthesis SuperMix kit (Beijing Transgen Biotech Co., Ltd.) according to the manufacturer's instructions. qPCR was conducted using the SYBR Green PCR Master Mix (Roche Diagnostics, Inc.) in a final reaction volume of $20 \mu \mathrm{l}$. Cyclic parameters of qPCR were as follows: Initial denaturation temperature at $95^{\circ} \mathrm{C}$ for $5 \mathrm{~min}$, followed by 40 cycles of $15 \mathrm{sec}$ denaturation at $95^{\circ} \mathrm{C}, 30 \mathrm{sec}$ annealing at $60^{\circ} \mathrm{C}$ and $45 \mathrm{sec}$ extension at $72^{\circ} \mathrm{C}$. Specific primer sequences are listed in Table I. The $\Delta \Delta \mathrm{Cq}$ method was used to calculate relative transcript levels (14). Relative gene expression was normalized to the expression levels of GAPDH and the experiment was repeated three times.

RNA interference. A549 cells were transfected with small interfering RNAs (siRNAs) using siTRAN 1.0 transfection reagent (cat. no. TT300001; OriGene Technologies, Inc.) (15). The siRNAs targeted to the CD44 (cat. no. SR319622) and NDRG1 (cat. no. SR307046) gene, and scrambled negative control siRNA (cat. no. SR30004) were purchased from OriGene Technologies, Inc. The sense sequence of siCD44 5'-GAACGAAUCCUGAAGACAUCU-3' and siNDRG1 5'-GCUGAAGCUCGUCAGUUCACCAUCC-3' were used in studies. The procedure was performed according to OriGeneproduct manuals. Briefly, $5 \mu \mathrm{l}$ siRNA and $20 \mu \mathrm{l}$ transfection reagent were mixed with $160 \mu$ l Opti-MEM I Reduced Serum Medium (Invitrogen; Thermo Fisher Scientific, Inc.). Then, the mixture was incubated at room temperature for $10 \mathrm{~min}$ before being added to 6 -well plates containing cells at confluence $60-70 \%$ for $48 \mathrm{~h}$. Cells were then subjected to further assays. Western blotting was performed to confirm CD44 and NDRG1 siRNA-mediated knockdown.

Internalization. The pathway by which A.fumigatus is internalized before infecting A549 cells was studied by a nystatin protection assay according to the method of Bao et al (16). A549 cells transfected with CD44 or NDRG1 siRNA were seeded in 24-well plates at a density of $2 \times 10^{4}$ cells/well $24 \mathrm{~h}$ prior to the experiment (Nest Biotech Co., Ltd.). The cells were incubated with A. fumigatus conidia at a MOI of 10 for $1 \mathrm{~h}$ at $37^{\circ} \mathrm{C}$ with $5 \% \mathrm{CO}_{2}$. Then, the cells were washed three times with PBST and incubated with nystatin in DMEM at $37^{\circ} \mathrm{C}$ for $4 \mathrm{~h}$. Subsequently, monolayer cells were washed three times with PBST and lysed by incubation in $0.25 \%$ Triton X-100 (BBI Life Sciences Corp.) diluted in PBS for $15 \mathrm{~min}$. The lysate was then serially diluted and plated on PDA plates (three replicate plates/well) and incubated at $37^{\circ} \mathrm{C}$ for 4 days before individual colony forming units were counted. The colonies were counted to determine the total intracellular conidia. The conidia internalization rate was calculated based on the formula: Conidia internalization rate $=$ the total intracellular conidia/the initial inoculum $\times 100 \%$. 
Determination of cytokine levels. A549 cells transfected with CD44 or NDRG1 siRNAwere seeded in 6-well plates at a density of $2 \times 10^{6}$ cells/well $24 \mathrm{~h}$ prior to the experiment (Nest Biotech Co., Ltd.). The cells were incubated with A.fumigatus conidia at a MOI of 10 for $12 \mathrm{~h}$ at $37^{\circ} \mathrm{C}$. The supernatants were collected and centrifuged at $1,500 \mathrm{x}$ g at $4^{\circ} \mathrm{C}$ for $15 \mathrm{~min}$ twice. IL-6 (cat. no. 88-7066-22; Thermo Fisher Scientific, Inc.), IL-8 (cat. no. 88-8086-22; Thermo Fisher Scientific, Inc.), TNF- $\alpha$ (cat. no. 88-7346-22; Thermo Fisher Scientific, Inc.) and IL-1 $\beta$ (cat. no. 88-7261-22; Thermo Fisher Scientific, Inc.) were assayed with ELISA kits according to the manufacturer's protocols.

Statistical analysis. All data are presented as the mean \pm SD of at least three replicate measurements. Mean values were compared using unpaired t-tests (two groups), followed by Bonferroni correction for multiple comparisons using one-way ANOVA and Tukey's post hoc. All statistical tests were performed using Prism software (v5.0; GraphPad Software, Inc.). P $<0.05$ was considered to indicate a statistically significant difference.

\section{Results}

Data analysis of the proteome of A549 cells infected with A. fumigatus. A total of 1,582 proteins were obtained after comparing the proteomes of A549 cells with or without A. fumigatus infection determined by iTRAQ labelling with the human protein database. A total of 111 differentially expressed proteins were identified, among which 18 were upregulated $(>1.5)$ and 93 were downregulated $(<0.75$; Table SI), including: NDRG1 (fold change,1.88; function, Rab GTPase binding); proteasome subunit $\alpha$ type-1 (1.74; threonine-type endopeptidase activity); SLK (1.69; protein serine/threonine kinase activity and adenyl ribonucleotide-binding ability); CD44 (0.47; primarily cytokine receptor activity and hyaluronic glucosaminidase activity), RNA-binding protein EWS isoform 1 (0.53; zinc ion binding). NDRG1 and CD44 were the most significantly upregulated and downregulated genes, respectively.

GO annotation and enrichment analyses of the differentially expressed proteins were performed from three perspectives: Biological process (BP), cellular component (CC) and molecular function (MF). Fig. 1 shows the top 20 most significantly enriched GO terms in the three categories (BP, CC and MF) determined by enrichment analysis. The aforementioned differentially expressed proteins were found to be involved in 1,169 biological processes $(\mathrm{P}<0.05$; Table SII). The BPs enriched in the largest number (n) of proteins were 'single-organism process' $(n=96)$, 'single-organism cellular process' $(n=91)$, 'cellular metabolic process' $(n=86)$, 'single-organism metabolic process' $(\mathrm{n}=66)$, "cellular component organization or biogenesis' $(\mathrm{n}=51)$ and 'response to stress' $(n=40)$. These differentially expressed proteins in A549 cells in the early stage of A.fumigatus infection compared with uninfected A549 cells are primarily related to biological processes, such as cell metabolism, synthesis and the cellular stress response. The differentially expressed proteins were primarily associated with binding-related functions, including 'protein binding' $(\mathrm{n}=75)$, 'organic cyclic compound binding' $(\mathrm{n}=55)$ and 'heterocyclic compound binding' $(\mathrm{n}=54)$, and catalytic functions, including 'hydrolase activity' $(\mathrm{n}=29)$, 'oxidoreductase activity' ( $\mathrm{n}=17)$ and 'transferase activity' $(n=13$; Table SIII). The majority of these proteins were located in 'membrane-bounded organelle' $(\mathrm{n}=105)$, 'intracellular organelle' $(n=100)$, 'intracellular membrane-bounded organelle' ( $n=98)$ and 'cytoplasm' ( $n=96)$, or 'intracellular organelle lumen' ( $\mathrm{n}=60$; Table SIV).

NDRG1, SLK and CD44 were the three proteins with the most significant differences in expression and primary function. These proteins are distributed in multiple parts of the cell and are involved in mainly biological processes, such as 'biological regulation' and 'response to stimulus'.

Results of KEGG pathway analysis. KEGG pathway enrichment analysis was performed for the 111 differentially expressed proteins using the KEGG pathway database. The results revealed 126 pathways primarily enriched in the differentially expressed proteins of A549 cells infected with A. fumigatus (Table SV). A total of 19 pathways $(\mathrm{P}<0.05)$ were obtained and the top 10 pathways were presented in Fig. 2. These pathways mainly concerned the 'TCA cycle', 'glyoxylate and dicarboxylate metabolism', 'pyruvate metabolism', 'carbon metabolism' and 'protein processing in endoplasmic reticulum'. The involved differential proteins had lower expression levels.

Interaction analysis of differentially expressed proteins. To further understand the protein-protein interactions between differentially expressed proteins, they were submitted to the STRING database (http://string.embl.de/) and subjected to analysis by Cytoscape software (http://www.cytoscape. org/). Fig. 3 illustrated the interaction network among the top 10 KEGG pathways significantly enriched in the involved proteins. As indicated in the figure, pathways related to metabolism, such as 'metabolic pathways', 'carbon metabolism' and 'central carbon metabolism in cancer', were at the centre of the interaction network. Citrate synthase, mitochondrial, isoform 1 of pyruvate dehydrogenase E1 component subunit $\beta$, mitochondrial, pyruvate dehydrogenase E1 component subunit $\alpha$, somatic form, mitochondrial isoform 2 precursor, dihydrolipoyl dehydrogenase, mitochondrial and malate dehydrogenase, mitochondrial were found to be involved in multiple metabolic pathways. These molecules are located mainly in the mitochondria.

Validation of the identified proteins and their quantification by western blotting and RT-qPCR. Western blotting and $\mathrm{qPCR}$ were employed to verify the accuracy of iTRAQ-mediated detection. NDRG1, SLK and CD44 levels in A549 cells infected with A. fumigatus determined by western blot analysis were consistent with the results of ITRAQ detection (Fig. 4A). The mRNA levels were consistent with their protein levels (Fig. 4B for protein and 4C for mRNA).

Knockdown of NDRG1 affects the internalization efficiency of A549 cells for A. fumigatus. To evaluate the role of NDRG1 and CD44 in the internalization of A. fumigatus by A549 cells, siRNAs were employed to knock down NDRG1 and $C D 44$ expression. The results demonstrated that the internalization efficiency was significantly lower following NDRG1-knockdown compared with the control group 


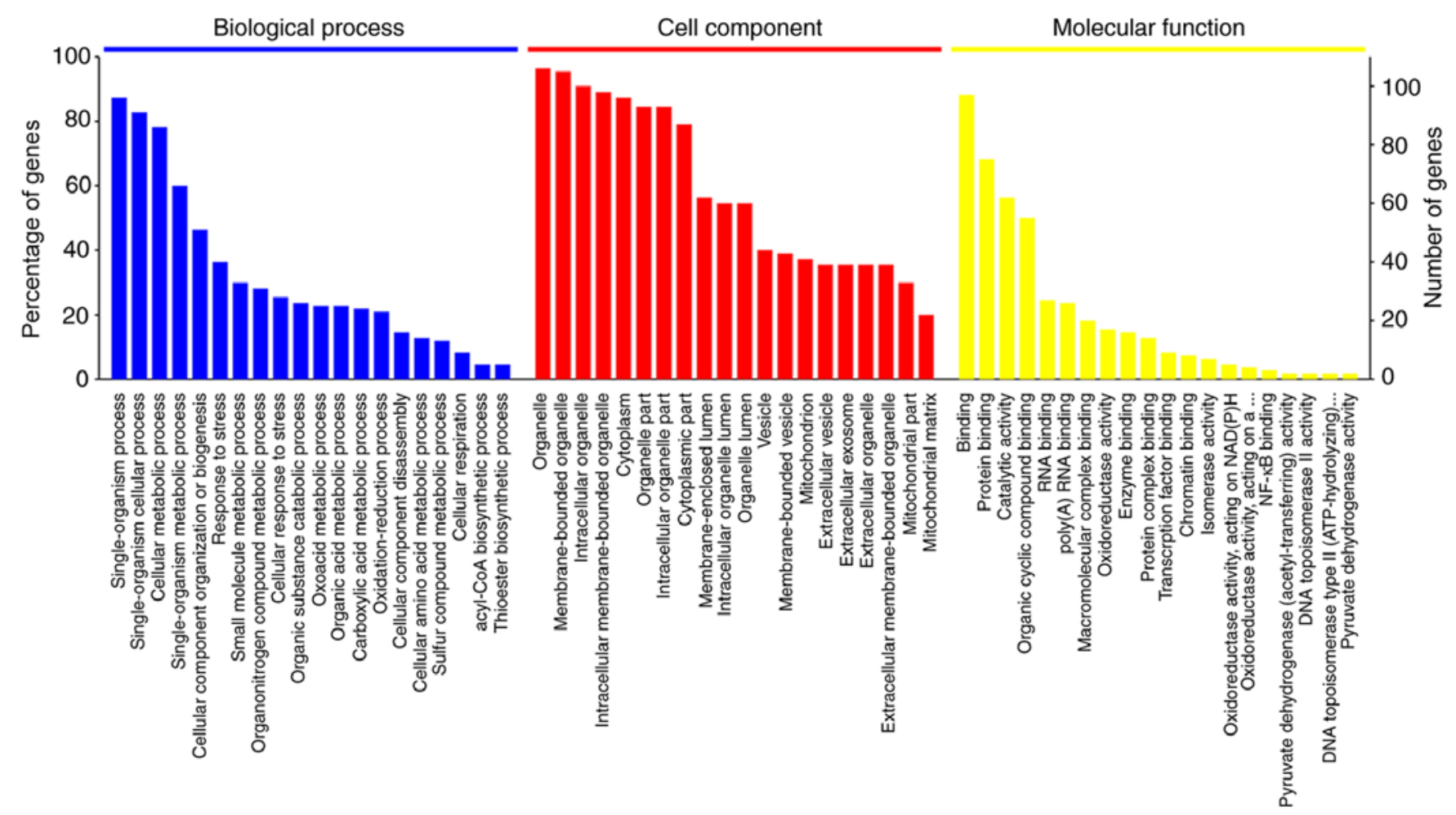

Figure 1. Top 20 most significantly enriched GO terms in differentially expressed proteins in A549 cells infected with Aspergillus fumigate conidia. Biological processes (blue), cell components (red) and molecular functions (yellow). The entries for each component are sorted from left to right according to the percentage of genes. The further to the left a GO term is, the more substantially it is enriched. The left and right vertical axes indicate the percentage and number of proteins corresponding to each entry, respectively. GO, Gene Ontology.

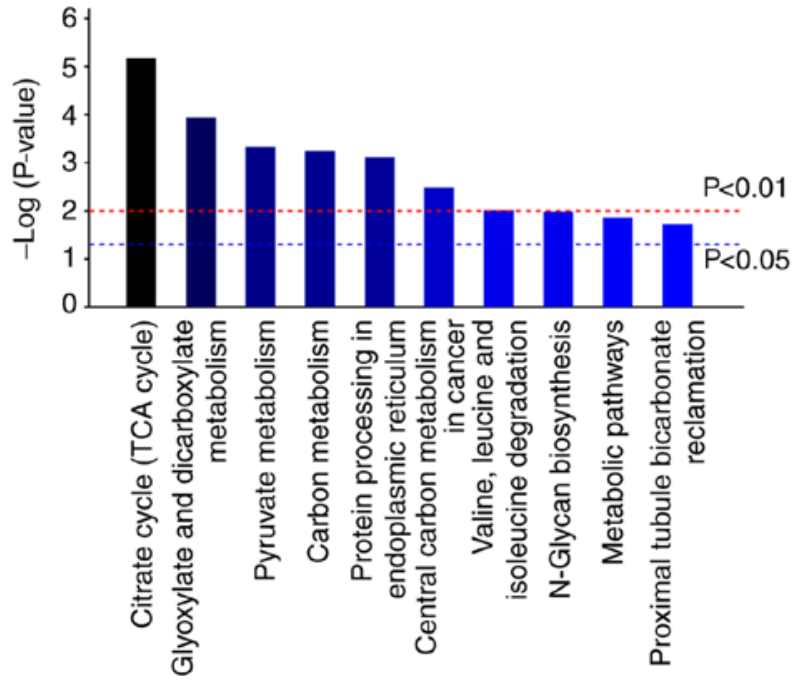

Figure 2. Top 10 most significantly enriched KEGG pathways in differentially expressed proteins in A549 cells infected with A.fumigatus compared with uninfected A549 cells. Each selected signaling pathway and its link to the KEGG database are shown on the abscissa. The ordinate indicates the negative logarithm of the $\mathrm{P}$-value to the base 2 . The red line indicates $\mathrm{P}<0.01$ and the blue line indicates $\mathrm{P}<0.05$. The regulatory path represented by each bar is significant only when the top of the bar is higher than the blue or red line. KEGG, Kyoto Encyclopedia of Genes and Genomes.

$(\mathrm{P}=0.0057$; Fig. 5A and $\mathrm{B})$. The internalization efficiency tended to increase following $C D 44$-knockdown, but this difference was not statistically significant $(\mathrm{P}=0.2655$; Fig. $5 \mathrm{C}$ and D).
Knockdown of CD44 reduces the secretion of $I L-8$ and $I L-6$ from A549 cells. To further investigate the effect of NDRG1 and $C D 44$ in A549 cells on the expression of cytokines in response to A.fumigatus infection, ELISA was performed. The results demonstrated that the presence of A.fumigatus led to the increased secretion of IL- 8 , IL- 6 , TNF- $\alpha$ and IL-1 $\beta$ from A549 cells. IL-6 $(\mathrm{P}=0.0461)$ and IL-8 $(\mathrm{P}=0.0014)$ were decreased to different extents after $C D 44$ knockdown, but there was no significant change in IL-1 $\beta$ and TNF- $\alpha$ levels. However, after NDRGl was knocked down, only IL-8 secretion was reduced $(\mathrm{P}=0.0096)$ and the expression of the other three cytokines examined remained constant (Fig. 6).

\section{Discussion}

The successful clearance of A.fumigatus conidia from the lungs depends on immune cells, such as monocytes/macrophages, neutrophils and airway epithelial cells, including bronchial epithelial cells and alveolar epithelial cells $(17,18)$. Additionally, epithelial cells serve an important role in clearing A. fumigatus (19). Studies have demonstrated that conidia adhere to the surface of A549 cells incubated with A.fumigatus conidia for $2 \mathrm{~h}$ with the assistance of RodA and CspA, surface components of the cell wall $(3,20)$. The internalization of the conidia by the cells at $4 \mathrm{~h}$ after infection is mediated by the activation of pattern recognition receptors, such as dectin-1, mannose receptors, or Toll-like receptors on endothelial cells (3). When co-incubated for $4 \mathrm{~h}$, the conidia begin to swell after being taken up by the endothelial cells to avoid being 


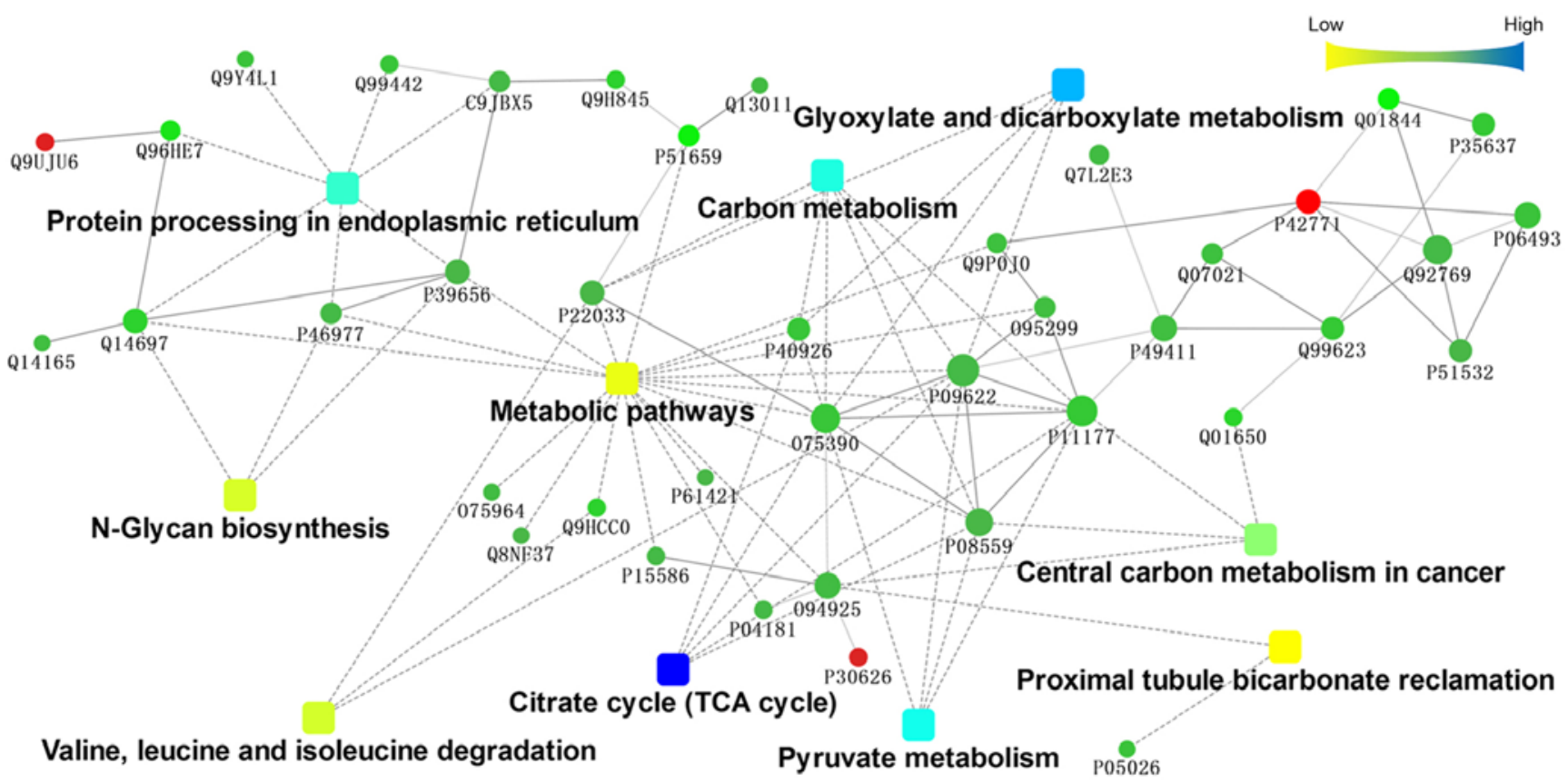

Figure 3. Analysis of the interactions between differentially expressed proteins in A549 cells infected with A. fumigatus conidia compared with uninfected A549 cells. The interaction analysis diagram illustrates the interaction network among the top 10 Kyoto Encyclopedia of Genes and Genomes pathways that were most significantly enriched in the involved proteins. The dots in the interaction map represent genes encoding the differentially expressed proteins (the red dots indicate proteins with upregulated expression and the green dots indicate proteins with downregulated expression). Squares indicate pathways enriched in differentially expressed genes (a gradual change in color from yellow to blue indicates a change in significance from low to high as shown by the gradient bar). A line from one dot to another dot indicates an interaction, a dotted line indicates an interaction that has not been experimentally verified and a solid line indicates an interaction that has been verified.

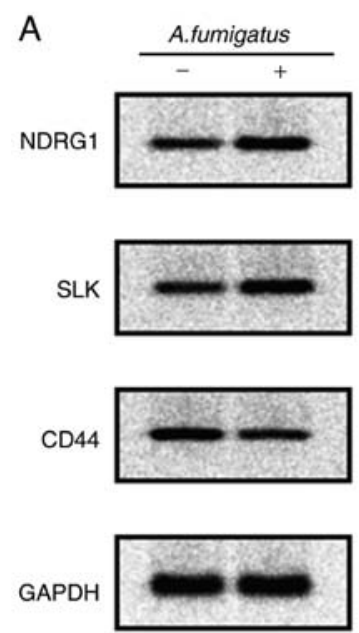

B
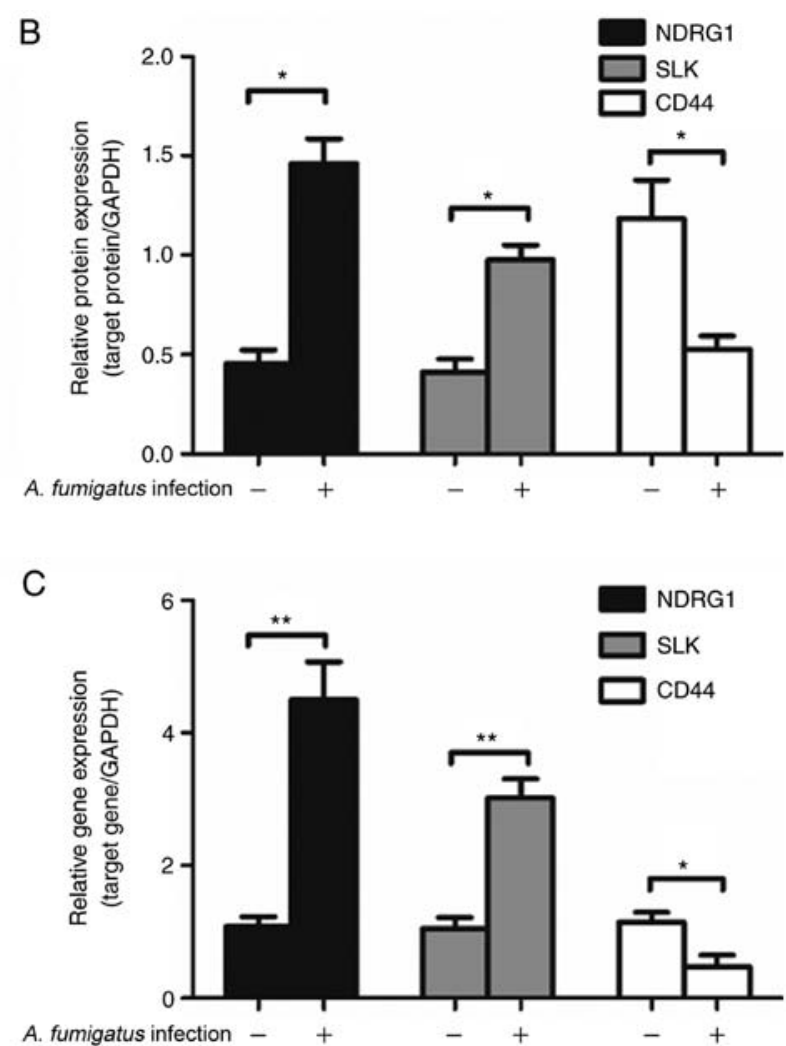

Figure 4. Determination of differentially expressed proteins in A549 cells infected with Aspergillus fumigatus conidia. (A) Conidia of A.fumigatus were co-incubated with A549 cells at a MOI of 10 for $6 \mathrm{~h}$ and the protein expression levels of NDRG1, SLK and CD44 proteins were analyzed by western blotting. (B) Data were expressed as the mean \pm standard error of the mean $(n=3)$ and analyzed using an independent sample $t$-test. "P<0.05. (C) Reverse transcription-quantitative PCR was used to analyze the expression levels of NDRG1, SLK and CD44 genes. Data are presented as the mean \pm standard error of the mean $(n=3)$ and analyzed using an independent sample $\mathrm{t}$-test; ${ }^{*} \mathrm{P}<0.05,{ }^{* * *} \mathrm{P}<0.01$. MOI, multiplicity of infection; NDRG1, N-myc downstream-regulated 1 ; SLK, STE20-like serine/threonine-protein kinase. 
A
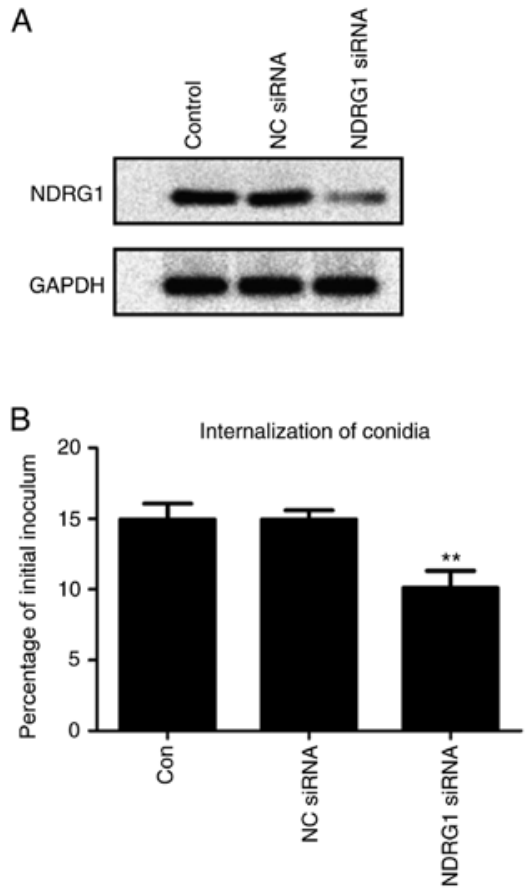

$\mathrm{C}$

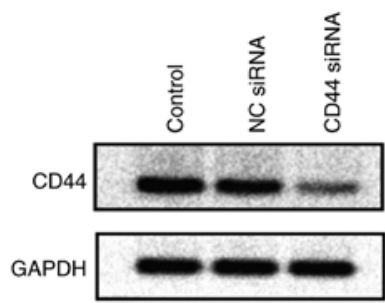

D

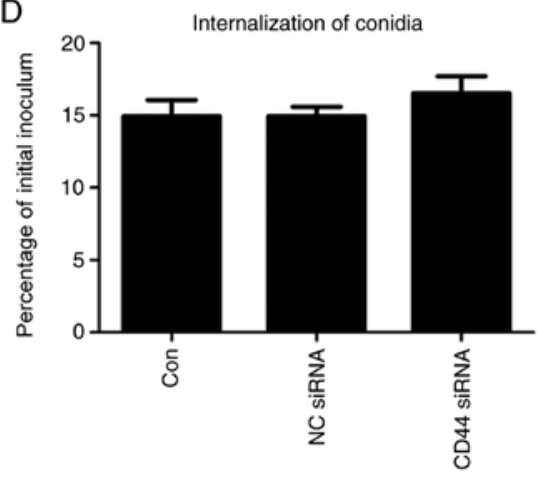

Figure 5. Changes in NDRG1 expression influence the internalization of A.fumigatus. After A549 cells were co-incubated with A.fumigatus conidia, A.fumigatus internalization was analyzed using a nystatin protection assay. A549 cells were transfected with a universal scrambled NC siRNA, (A) NDRG1-specific siRNA, or (C) CD44-specific siRNA. After $48 \mathrm{~h}$, the cells were infected with A. fumigatus conidia at a multiplicity of infection of 10. Internalization of A. fumigatus was analyzed by nystatin protection assay for (B) NDRG1 and (D) CD44 siRNA. Data are presented as the mean \pm standard error of the mean (n=6). One-way ANOVA and Tukey's post hoc multiple comparison tests were used to detect changes between individual groups. ${ }^{* *} \mathrm{P}<0.01$ vs. control group. Western blotting was used to detect the expression levels of NDRG1 and CD44 in A549 cell lysates. A. fumigatus, Aspergillus fumigatus; Con, control; NDRG1, N-myc downstream-regulated 1; NC, negative control; siRNA, small interfering RNA.
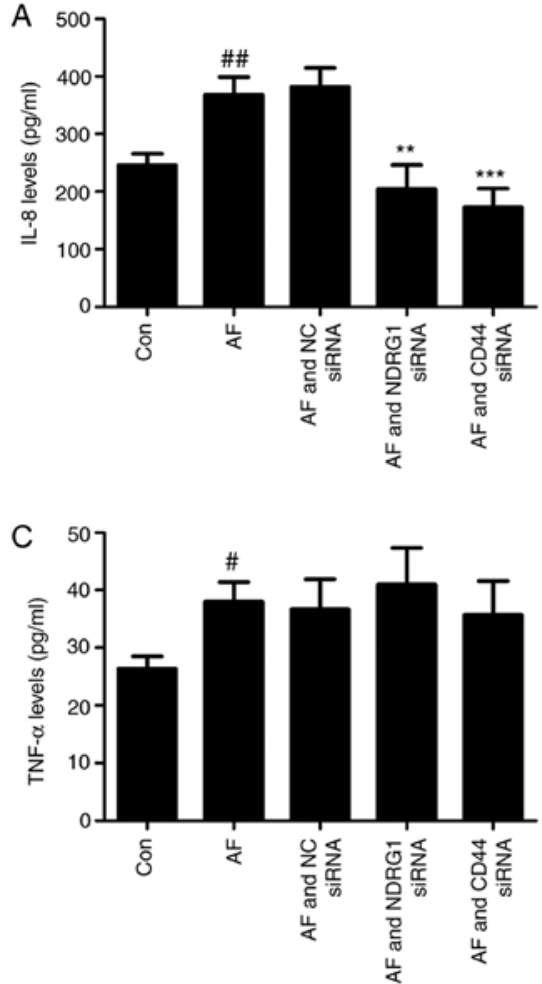
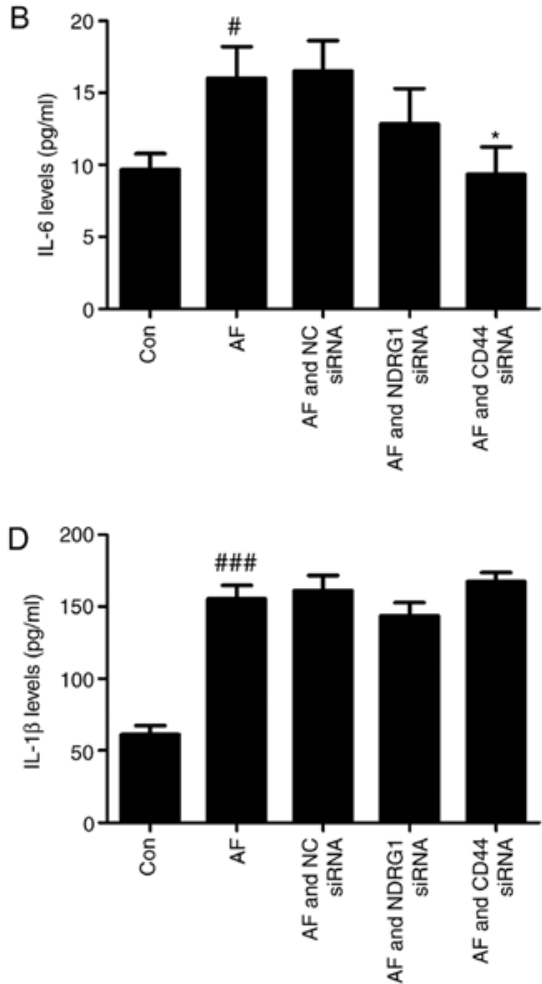

Figure 6. NDRG1 and CD44 knockdown affects the expression levels of cytokines. A549 cells were incubated with A. fumigatus conidia at a multiplicity of infection of 10 for a total of $12 \mathrm{~h}$. Differences in the expression levels of (A) IL-8, (B) IL-6, (C) TNF- $\alpha$ and (D) IL-1 $\beta$ in the cell supernatants of the uninfected group (Con), the A.fumigatus conidia-infected group (AF), the NC siRNA-transfected group (AF and NC siRNA), the NDRG1-knockdown and A. fumigatus conidia-infected group (AF and NDRG1 siRNA) and the CD44-knockdown and A. fumigatus conidia-infected group (AF and CD44 siRNA) were determined. Data are presented as the mean \pm standard error of the mean $(\mathrm{n}=6)$. Data analysis was performed using one-way ANOVA and Tukey's post hoc multiple comparison tests. ${ }^{\mathrm{P}}<0.05,{ }^{* *} \mathrm{P}<0.01$ and ${ }^{* * * *} \mathrm{P}<0.001$ vs. AF group; ${ }^{\#} \mathrm{P}<0.05,{ }^{\# \#} \mathrm{P}<0.01$ and ${ }^{\# \# "} \mathrm{P}<0.001$ vs. Control group. A. fumigatus, Aspergillus fumigatus; IL, interleukin; TNF- $\alpha$, tumor necrosis factor $\alpha$; NC, negative control; siRNA, small interfering RNA. 
killed by immune cells and to ensure their survival (21). After 6-8 $\mathrm{h}$ post-infection, the conidia on the epithelial cells are used as 'kindling' that eventually stimulates germination and the formation of germ tubes (20), although the survival rate of conidia on the epithelial cells decreases sharply. In the present study, the iTRAQ method was used to observe changes in the proteome of A549 cells following infection with A.fumigatus conidia for $6 \mathrm{~h}$ and the molecular mechanism of A.fumigatus conidia infection, and excluded the effects of conidia swelling and the germination of germ tubes.

GO enrichment and KEGG pathway analyses revealed that most differentially expressed proteins were involved in basic biological processes, including cell energy metabolism, substance metabolism and cell synthesis. The expression levels of some proteins related to cytoskeletal rearrangement, such as stathmin (fold change of 1.67), CAP-Gly domain containing linker protein 1 (1.66), protein phosphatase 1 regulatory subunit $12 \mathrm{~A}$ (1.54), kinesin family member $2 \mathrm{~A}$ (0.71) and cyclin-dependent kinase 1 (0.69), changed with A. fumigatus infection. These molecules are mainly involved in the upstream regulatory process. Chen et al (21) analyzed changes in the transcriptome of A549 cells following infection with A. fumigatus conidia for $8 \mathrm{~h}$ and reported that expression of the molecules leukocytespecific transcript 1, activity-regulated cytoskeleton-associated protein, early growth response protein (EGR)1 and EGR4, which are involved in the rearrangement of the cytoskeleton, change when A549 cells are infected with A. fumigatus conidia for $8 \mathrm{~h}$. The results of the present study indicated changes in molecular regulation at $6 \mathrm{~h}$ following infection in preparation for endocytosis.

The molecules cytochrome b-245 light chain (0.7), cathepsin S (0.7) and complement component $1 \mathrm{Q}$ subcomponent-binding protein (0.7), which may participate in the processing of endocytosed antigens, reactive oxygen species production, the innate immune response, and antigen processing and presentation, are suppressed by A. fumigatus infection (Tables SI-SIV). Notably, the above proteins were associated with Toll-like receptor signaling pathways and RIG-I-like receptor signaling pathways, which indicated that these pathways were involved in A.fumigatus infection. The findings of the present study were consistent with the transcriptome and metabolome of A.fumigatus-infected human dendritic cells (22). Thus, the function of non- $C$ type lectin-like pattern recognition receptors deserves more attention in studies of the interaction between A.fumigatus conidia and lung epithelial cells.

NDRG1, a member of the NDRG family (NDRG 1-4), is expressed in epithelial cells (23). NDRG1 is involved in multiple cell biological processes, such as cell proliferation, differentiation and development (24). However, the role of NDRG1 in viral infection is ambiguous. NDRG1 limits the life cycle of hepatitis $C$ virus (25) but promotes the replication of influenza A virus (23). The present study identified that the infection of A549 cells with A. fumigatus significantly increased the protein expression of NDRG1, which serves an important role in maintaining the integrity of the lung epithelial cell barrier (26). However, whether elevated NDRG1 expression on epithelial cells is helpful for A.fumigatus conidia invasion or the body's defense remains to be elucidated. The internalization efficiency was reduced when the NDRGl gene was silenced, but NDRG1 silencing had little effect on the expression levels of cytokines. This indicated that NDRG1 may have a positive regulatory effect on the rearrangement of cytoskeletal proteins, thereby promoting A. fumigatus conidia internalization.

CD44 is a transmembrane adhesion molecule with has hyaluronic glucosaminidase activity and cytokine receptor activity. It is widely expressed in a number of cells and can recognize a variety of ligands, including hyaluronan (HA), collagen, laminin, fibronectin, osteopontin and other extracellular proteins involved in cell adhesion, growth, proliferation and motility (27-29). Studies have demonstrated that CD44 can promote or inhibit microbial infection depending on the type of microorganism and the initial infection site $(30,31)$. CD44-deficient mice have prolonged survival rates but elevated lung inflammation compared with wild-type mice following infection with a lethal dose of Klebsiella pneumoniae by intranasal administration (31). However, the effect of Streptococcus equi subsp. Zooepidemicus infection is in contrast to that of Klebsiella pneumoniae infection (31). CD44-knockout mice survive longer and have a lower fungal burden in the brain and cerebrospinal fluid than wild-type mice following lateral venule injection of Cryptococcus neoformans (32). This may be because HA on the surface of Cryptococcus neoformans binds CD44 on the surface of human brain microvascular endothelial cells (33). It is known that CD44 promotes cell adhesion and invasion $(32,33)$. In the present study, the inhibition of CD44 expression may be caused by the absence of CD44-related ligands on the cell wall surface of A. fumigatus. Qadri et al (34) demonstrated that $\mathrm{CD} 44-\mathrm{knockdown}$ reduces $\mathrm{NF}-\kappa \mathrm{B}$ nuclear translocation and downstream proinflammatory cytokines production. Therefore, the present study assayed the expression levels of proinflammatory cytokines following transfection with CD44 siRNA. This demonstrated that the expression levels of IL- 6 and IL- 8 were reduced, which indicated that A. fumigatus conidia could inhibit the inflammatory reaction in epithelial cells via CD44. However, CD44 does not affect the internalization of A. fumigatus conidia, which is in contrast to Cryptococcus neoformans (32). This phenomenon might be related to the surface content of the fungal cell wall. The study of CD44 and its role in the extracellular matrix in fungal infection would be of great interest.

In summary, to the best of our knowledge, proteomic data on the interaction between A. fumigatus conidia and A549 alveolar epithelial cells have been acquired for the first time and a number of differentially expressed proteins, including NDRG1 and CD44, were identified. In addition, NDRG1 and CD44 were found to affect endocytosis and cytokine expression in A549 cells, respectively, and these proteins may serve as diagnostic targets and may be involved in treatment regimens for invasive pulmonary aspergillosis.

\section{Acknowledgements}

The authors would like to thank Professor Koji Yokoyama, from Medical Mycology Research Center of Chiba University (Chiba, Japan), for providing the A. fumigatus strain for the current research. 


\section{Funding}

The present study was supported in part by the National Natural Science Foundation of China (grant no. 81271802) and the National Science and Technology Major Project of the Ministry of Science and Technology of China (grant no. 2013ZX10004612-006).

\section{Availability of data and materials}

All data generated or analyzed during this study are included in this published article.

\section{Authors' contributions}

XZ performed the laboratory experiments and drafted the manuscript. DH analyzed the experimental data and revised the manuscript. SG and YW participated in laboratory experiments. LW designed the project and supervised the present study. All authors read and approved the final manuscript.

\section{Ethics approval and consent to participate}

Not applicable.

\section{Patient consent for publication}

Not applicable.

\section{Competing interests}

The authors declare that they have no competing interests.

\section{References}

1. Latgé JP: The pathobiology of Aspergillus fumigatus. Trends Microbiol 9: 382-389, 2001.

2. Ogorman CM: Airborne Aspergillus fumigatus conidia: A risk factor for aspergillosis. Fungal Biol Rev 25: 151-157, 2011.

3. Croft CA, Culibrk L, Moore MM and Tebbutt SJ: Interactions of Aspergillus fumigatus Conidia with airway epithelial cells: A critical review. Front Microbiol 7: 472, 2016.

4. Bassetti M, Garnacho-Montero J, Calandra T, Kullberg B, Dimopoulos G, Azoulay E, Chakrabarti A, Kett D, Leon C, Ostrosky-Zeichner L, et al: Intensive care medicine research agenda on invasive fungal infection in critically ill patients. Intensive Care Med 43: 1225-1238, 2017.

5. Toor A, Culibrk L, Singhera GK, Moon KM, Prudova A, Foster LJ, Moore MM, Dorscheid DR and Tebbutt SJ: Transcriptomic and proteomic host response to Aspergillus fumigatus conidia in an air-liquid interface model of human bronchial epithelium. PLoS One 13: e0209652, 2018.

6. Jia X, Chen F, Pan W, Yu R, Tian S, Han G, Fang H, Wang S, Zhao J, Li X, et al: Gliotoxin promotes Aspergillus fumigatus internalization into type II human pneumocyte A549 cells by inducing host phospholipase D activation. Microbes Infect 16: 491-501, 2014.

7. Paris S, Boisvieux-Ulrich E, Crestani B, Houcine O, Taramelli D, Lombardi L and Latgé JP: Internalization of Aspergillus fumigatus conidia by epithelial and endothelial cells. Infect Immun 65 : 1510-1514, 1997.

8. Ye F, Zhang H, Yang YX, Hu HD, Sze SK, Meng W, Qian J, Ren H, Yang BL, Luo MY, et al: Comparative proteome analysis of 3T3-L1 adipocyte differentiation using iTRAQ-coupled 2D LC-MS/MS. J Cell Biochem 112: 3002-3014, 2011.

9. Vuong NQ, Goegan P, Mohottalage S, Breznan D, Ariganello M, Williams A, Elisma F, Karthikeyan S, Vincent R and Kumarathasan P: Proteomic changes in human lung epithelial cells (A549) in response to carbon black and titanium dioxide exposures. J Proteomics 149: 53-63, 2016.
10. She X, Zhang P, Gao Y, Zhang L, Wang Q, Chen H, Calderone R, Liu W and Li D: A mitochondrial proteomics view of complex I deficiency in Candida albicans. Mitochondrion 38: 48-57, 2018.

11. Pizzatti L, Binato R, Cofre J, Gomes BE, Dobbin J, Haussmann ME, D'Azambuja D, Bouzas LF and Abdelhay E: SUZ12 is a candidate target of the non-canonical WNT pathway in the progression of chronic myeloid leukemia. Genes Chromosomes Cancer 49: 107-118, 2010.

12. Zhang M, Cheng ST, Wang HY, Wu JH, Luo YM, Wang Q, Wang FX and Xia GX: iTRAQ-based proteomic analysis of defence responses triggered by the necrotrophic pathogen Rhizoctonia solani in cotton. J Proteomics 152: 226-235, 2017.

13. Shannon P, Markiel A, Ozier O, Baliga NS, Wang JT, Ramage D, Amin N, Schwikowski B and Ideker T: Cytoscape: A software environment for integrated models of biomolecular interaction networks. Genome Res 13: 2498-2504, 2003.

14. Livak KJ and Schmittgen TD: Analysis of relative gene expression data using real-time quantitative PCR and the 2(-Delta Delta C(T)) method. Methods 25: 402-408, 2001.

15. Li W, Cohen A, Sun Y, Squires J, Braas D, Graeber TG, Du L, $\mathrm{Li} \mathrm{G}, \mathrm{Li} \mathrm{Z}, \mathrm{Xu}$ X, et al: The role of CD44 in glucose metabolism in prostatic small cell neuroendocrine carcinoma. Mol Cancer Res 14: 344-353, 2016

16. Bao Z, Han X, Chen F, Jia X, Zhao J, Zhang C, Yong C, Tian S, Zhou $X$ and Han L: Evidence for the involvement of cofilin in Aspergillus fumigatus internalization into type II alveolar epithelial cells. BMC Microbiol 15: 161, 2015.

17. Seddigh $P, B r a c h t T$, Molinier-Frenkel V, Castellano F, Kniemeyer O, Schuster M, Weski J, Hasenberg A, Kraus A, Poschet G, et al: Quantitative analysis of proteome modulations in alveolar epithelial type II cells in response to pulmonary Aspergillus fumigatus Infection. Mol Cell Proteomics 16: 2184-2198, 2017.

18. Rabinovitch M: Professional and non-professional phagocytes: An introduction. Trends Cell Biol 5: 85-87, 1995.

19. Crapo JD, Barry BE, Gehr P, Bachofen M and Weibel ER: Cell number and cell characteristics of the normal human lung. Am Rev Respir Dis 126: 332-337, 1982.

20. Escobar N, Ordonez SR, Wosten HA, Haas PJ, de Cock H and Haagsman HP: Hide, keep quiet, and keep low: Properties that make Aspergillus fumigatus a successful lung pathogen. Front Microbiol 7: 438, 2016.

21. Chen F, Zhang C, Jia X, Wang S, Wang J, Chen Y, Zhao J, Tian S, Han X and Han L: Transcriptome profiles of human lung epithelial cells A549 interacting with Aspergillus fumigatus by RNA-Seq. PLoS One 10: e0135720, 2015.

22. Srivastava M, Bencurova E, Gupta SK, Weiss E, Loffler J and Dandekar T: Aspergillus fumigatus challenged by human dendritic cells: Metabolic and regulatory pathway responses testify a tight battle. Front Cell Infect Microbiol 9: 168, 2019.

23. Chen L, Xing C, Ma G, Luo J, Su W, Li M, Shi Q and He H: $\mathrm{N}$-myc downstream-regulated gene 1 facilitates influenza $\mathrm{A}$ virus replication by suppressing canonical NF-kappaB signaling. Virus Res 252: 22-28, 2018.

24. Melotte V, Qu X, Ongenaert M, van Criekinge W, de Bruïne AP, Baldwin HS and van Engeland M: The N-myc downstream regulated gene (NDRG) family: Diverse functions, multiple applications. FASEB J 24: 4153-4166, 2010.

25. Schweitzer CJ, Zhang F, Boyer A, Valdez K, Cam M and Liang TJ: N-Myc downstream-regulated gene 1 restricts hepatitis $\mathrm{C}$ virus propagation by regulating lipid droplet biogenesis and viral assembly. J Virol 92: e01166-1792, 2018.

26. Gon Y, Maruoka S, Kishi H, Kozu Y, Kazumichi K, Nomura Y, Takeshita I, Oshima T and Hashimoto S: NDRG1 is important to maintain the integrity of airway epithelial barrier through claudin-9 expression. Cell Biol Int 41: 716-725, 2017.

27. Ouhtit A, Rizeq B, Saleh HA, Rahman MM and Zayed H: Novel CD44-downstream signaling pathways mediating breast tumor invasion. Int J Biol Sci 14: 1782-1790, 2018.

28. Naor D, Nedvetzki S, Golan I, Melnik L and Faitelson Y: CD44 in cancer. Crit Rev Clin Lab Sci 39: 527-579, 2002.

29. Babasola O, Rees-Milton KJ, Bebe S, Wang J and Anastassiades TP: Chemically modified $\mathrm{N}$-acylated hyaluronan fragments modulate proinflammatory cytokine production by stimulated human macrophages. J Biol Chem 289: 24779-24791, 2014.

30. Fu Q, Wei Z, Xiao P, Chen Y and Liu X: CD44 enhances macrophage phagocytosis and plays a protective role in Streptococcus equi subsp. zooepidemicus infection. Vet Microbiol 198: 121-126, 2017. 
31. van der Windt GJ, Florquin S, de Vos AF, van't Veer C, Queiroz KC, Liang J, Jiang D, Noble PW and van der Poll T: CD44 deficiency is associated with increased bacterial clearance but enhanced lung inflammation during Gram-negative pneumonia. Am J Pathol 177: 2483-2494, 2010.

32. Jong $\mathrm{A}$, Wu CH, Gonzales-Gomez I, Kwon-Chung KJ, Chang YC, Tseng HK, Cho WL and Huang SH: Hyaluronic acid receptor CD44 deficiency is associated with decreased Cryptococcus neoformans brain infection. J Biol Chem 287: 15298-15306, 2012

33. Chang YC, Stins MF, McCaffery MJ, Miller GF, Pare DR, Dam T, Paul-Satyaseela M, Kim KS and Kwon-Chung KJ: Cryptococcal yeast cells invade the central nervous system via transcellular penetration of the blood-brain barrier. Infect Immun 72: 4985-4995, 2004.
34. Qadri M, Almadani S, Jay GD and Elsaid KA: Role of CD44 in regulating TLR2 activation of human macrophages and downstream expression of proinflammatory cytokines. J Immun 200: 758-767, 2018.

This work is licensed under a Creative Commons Attribution-NonCommercial-NoDerivatives 4.0 International (CC BY-NC-ND 4.0) License. 